\title{
WEAK ANTILOCALIZATION IN QUANTUM WELLS
}

\author{
W. Knap, C. Skierbiszewski* , E. Litwin-Staszewska*, F. Kobbi,
} A. ZDUNIAK ${ }^{\dagger}$, J.L. ROBERT

Groupe d'Etude des Semiconducteurs, Université Montpellier II

Place E. Bataillon, 34095 Montpellier-Cedex 05, France

$$
\text { G.E. PIKUS }
$$

A.F. Ioffe Physicotechnical Institute, Russian Academy of Sciences

St Petersbourg, Russia

\section{S.V. IORDANSKII}

Landau Institute for Theoretical Physics, 117940 Moscow, Russia

$$
\text { V. MOSSER }
$$

Schlumberger Montrouge Recherche 50

Avenue Jean Jaurés, B.P. 620-05, 92542 Montrouge, France

\section{AND K. ZeKENTES}

Forth Institute of Electronic Structure and Laser P.O. Box 1527, Heraklion, 71110 - Crete, Greece

Spin relaxation in degenerated two-dimensional (2D) electron gas is studied by measurements of the magnetic field dependence of the weak antilocalization corrections to the conductivity in GaInAs quantum wells. Consistent quantitative (up to order of magnitude) description of weak antilocalization data on $\mathrm{GaAs}$ like heterojunctions and quantum wells was obtained. Our results show that spin precession around the effective magnetic field direction as described by the Dyakonov-Perel model is the main spin relaxation mechanism in degenerated $2 \mathrm{D}$ electron gas in semiconductors with no inversion symmetry.

PACS numbers: 72.15.Lh, 73.20.Fz, 72.15.Rn

*On leave from the High Pressure Research Center, Polish Academy of Sciences, 01-142 Warszawa, Poland.

tOn leave from Department of Experimental Physics, Warsaw University, 00-681 Warszawa, Poland. 


\section{Quantum corrections to the conductivity}

Weak localization comes from the constructive interference of two time reversal paths of electrons scattered in the disordered systems. It leads to the quantum reduction of the conductance. However, in the presence of strong spin scattering, the two time reversal paths can give rise to destructive interference and enhance the conductance. This phenomenon is called weak antilocalization. Magnetic field destroys both the spatial and the spin interferences. Therefore, with increasing magnetic field one can observe low field positive magnetoresistance (weak antilocalization feature) followed by negative magnetoresistance (weak localization feature).

In this section we present the main results of the theory of quantum corrections to the conductivity due to weak antilocalization. More detailed derivations are presented in Ref. [1]. The quantum corrections to the conductivity $\Delta \sigma(B)$ can be expressed by the formula

$$
\begin{aligned}
& \Delta \sigma(B)=-\frac{e^{2}}{\pi h}\left[\psi\left(\frac{1}{2}+\frac{H_{\mathrm{tr}}}{B}\right)-\psi\left(\frac{1}{2}+\frac{H_{2}}{B}\right)+\frac{1}{2} \psi\left(\frac{1}{2}+\frac{H_{\phi}}{B}\right)\right. \\
& \left.-\frac{1}{2} \psi\left(\frac{1}{2}+\frac{H_{4}}{B}\right)\right],
\end{aligned}
$$

where

$$
\Delta \sigma(0)=-\frac{e^{2}}{\pi h}\left[\ln \left(\frac{H_{\mathrm{tr}}}{H_{2}}\right)+\frac{1}{2} \ln \left(\frac{H_{\varphi}}{H_{4}}\right)\right],
$$

$B$ is the magnetic field, $\psi(x)$ is the digamma function defined by

$$
\psi(x)=\sum_{n=1}^{\infty} \frac{x}{n(x+n)}, \quad H_{2}=H_{\varphi}+H_{\mathrm{so}}, \quad H_{4}=H_{\varphi}+2 H_{\mathrm{so}} .
$$

The characteristic transport $H_{\mathrm{tr}}$, phase $H_{\varphi}$ and $H_{\mathrm{so}}$ magnetic fields are related to the corresponding relaxation times $\tau_{\mathrm{tr}}, \tau_{\varphi}, \tau_{s x}$ by

$$
H_{\mathrm{tr}}=\frac{\alpha}{\tau_{1}}, \quad H_{\varphi}=\frac{\alpha}{\tau_{\varphi}}, \quad H_{\mathrm{so}}=\frac{\alpha}{\tau_{s x}}, \quad \alpha=\frac{\hbar}{4 e D},
$$

where $D$ is the diffusion constant. Formula (1) is as Hikami-Larkin-Nagaoka formula [2] but the definition of $H_{\text {so }}$ is different by a factor 2 .

In the Dyakonov-Perel mechanism the spin relaxation is due to the spin precession around the effective magnetic field. This field depends on the electron wave vector and changes due to scattering. For $2 \mathrm{D}$ electron gas $\tau_{s x}^{-1}$, the spin relaxation rate of the $x$ component of the spin, can be written [1] as a sum of two contributions which corresponds to two spin precessions with frequencies $\omega_{1}, \omega_{3}$ and corresponding relaxation times $\tau_{1}$ and $\tau_{3}$ :

$$
\frac{1}{\tau_{s x}}=\frac{1}{2}\left(\omega_{1}^{2} \tau_{1}+\omega_{3}^{2} \tau_{3}\right) .
$$

$\tau_{1}, \tau_{3}$ are defined by

$$
\frac{1}{\tau_{n}}=\int W(\theta)(1-\cos n \theta) \mathrm{d} \theta
$$


where $W(\theta)$ is the probability of the change of the electron wave vector direction by the angle $\theta$ in unit time due to elastic scattering. Precession frequencies can be expressed as

$$
\begin{aligned}
& \omega_{1}^{2}=\frac{4 a_{42}^{2}}{\hbar^{2}} k_{\mathrm{p}}^{2}\left(\left\langle k_{z}^{2}\right\rangle-\frac{k_{\mathrm{p}}^{2}}{4}\right)^{2}, \\
& \omega_{3}^{2}=\frac{4 a_{42}^{2}}{\hbar^{2}} \frac{k_{\mathrm{p}}^{6}}{16},
\end{aligned}
$$

$k_{\mathrm{p}}$ and $k_{z}$ are respectively in-plane and $z$ component of the wave vector, $a_{42}$ is the band structure constant [3]. Formal derivation of the relation (3) can be found in Ref. [1].

Using relations (2) and (3), replacing $k_{\mathrm{p}}$ by $k_{\mathrm{f}}$ because for degenerated electron gas only electrons at the Fermi surface play role and expressing the diffusion constant $D$ for the degenerate 2D gas as

$$
D=\frac{1}{2}\left(\frac{\hbar k_{\mathrm{f}}}{m^{*}}\right)^{2} \tau_{1}
$$

we finally get the expression for the characteristic spin relaxation field $H_{\text {so }}$ which is usually determined in the weak antilocalization experiments

$$
H_{\mathrm{so}}\left(N_{\mathrm{s}}\right)=\frac{\eta}{(2 \pi)^{2}}\left(\frac{1+\tau_{3} / \tau_{1}}{2} k_{\mathrm{f}}^{4}-4\left\langle k_{z}^{2}\right\rangle k_{\mathrm{f}}^{2}+8\left\langle k_{z}^{2}\right\rangle^{2}\right)
$$

where $k_{\mathrm{f}}^{2}=2 \pi N_{\mathrm{s}}$.

Coefficient

$$
\eta=\frac{\pi m^{* 2}}{4 e \hbar^{3}} a_{42}^{2}
$$

was chosen to have the same meaning as in Ref. [4].

\section{Experimental}

Two AlGaAs/InGaAs/GaAs pseudomorphic quantum wells were studied. They were grown by the molecular beam epitaxy technique. The layer sequence of the structure were of the standard HEMT type [5]. Both samples were $\delta$-doped with Si (doping density $N_{\delta}=2.5 \times 10^{12} \mathrm{~cm}^{-2}$ ).

In order to study the behavior of the structures as a function of $N_{\mathrm{s}}$, the metastable properties of the DX Si centers present in AlGaAs-layer were used. This allowed to tune carrier density from $1.06 \times 10^{12} / \mathrm{cm}^{2}$ to $1.29 \times 10^{12} / \mathrm{cm}^{2}$ for sample A and from $1.52 \times 10^{12} / \mathrm{cm}^{2}$ to $2.05 \times 10^{12} / \mathrm{cm}^{2}$ for sample $B$.

We used a system of two superconducting coils $(8 \mathrm{~T} / 8 \mathrm{~T})$ placed in the same cryostat - the scattered field of one coil was compensated by another one allowing to get good tunability and uniformity of the magnetic field in the sample space. The same system was earlier used to study cyclotron emission from different semiconductors systems and is described in more detail in Ref. [6]. 


\section{Results and discussion}

Quantitative analysis of the data was performed on the basis of formula (1). $H_{\text {tr }}$ was determined by transport measurements of carrier density and mobility. Usually parameters $H_{\text {so }}$ and $H_{\varphi}$ should be determined by fitting formula (1) to the experimental results. One can improve this by fitting not only the conductivity but also its first derivative. Figure 1 shows example of the data and its derivative and results of fit.
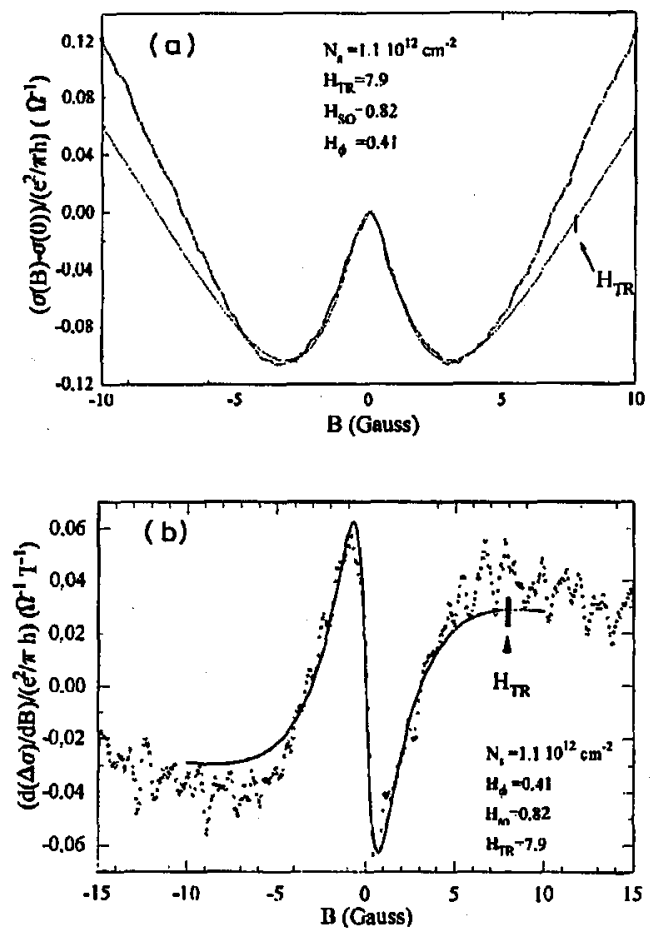

Fig. 1. Example of fit of formula (1) (a) and its first derivative (b) to the experimental results.

In Fig. 2 dependence of the $H_{\text {so }}$ on the carrier density is showed. Continuous lines are results of the calculation according to (4). For value $a_{42}$ we took $24 \mathrm{eV} \AA^{3}$ as found in measurements of spin relaxation by optical orientation [7] and band-structure calculations [3] for GaAs. The effective mass $m^{*}$ was set at $0.067 m_{0}$ as determined by cyclotron resonance emission measurements performed on the same samples [5]. Value of $\left\langle k_{z}^{2}\right\rangle$ was obtained from self-consistent Poisson-Schrödinger calculations.

Special feature of the $H_{\mathrm{so}}\left(N_{\mathrm{s}}\right)$ dependence for quantum wells is that $H_{\mathrm{so}}$ starts from important value for $N_{\mathrm{s}}=0$ and then decreases reaching minimum. Because the experimentally determined value of $H_{\text {so }}$ increases with $N_{\mathbf{s}}$, beginning from about $1.2 \times 10^{12} / \mathrm{cm}^{2}$ one can state that at least for higher concentrations 


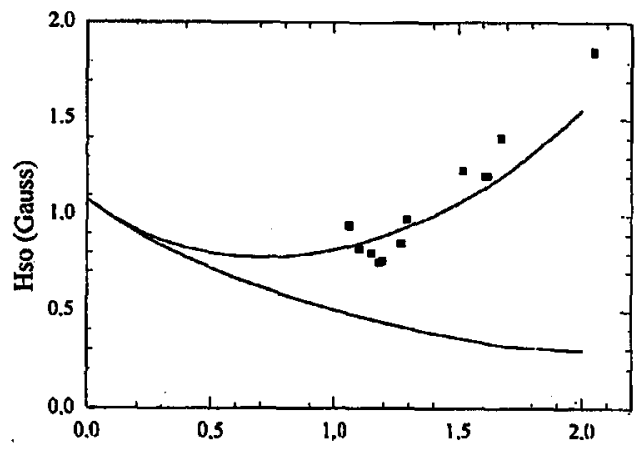

Fig. 2. Quantum well: $H_{\mathrm{so}}$ as a function of the carrier density $N_{\mathrm{s}}$ theory and experiment. Theoretical results are presented in two limit cases: short range scattering $\left(\tau_{1}=\tau_{3}\right.$, upper curve) and long range scattering ( $\tau_{1}=9 \tau_{3}$, lower curve).

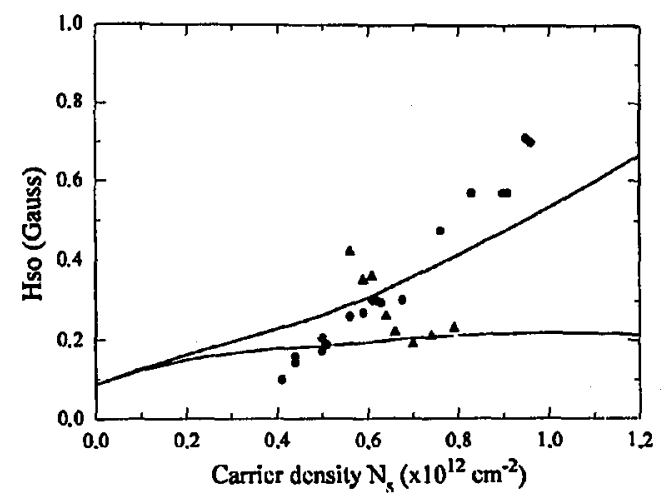

Fig. 3. Heterojunction: $H_{\mathrm{so}}$ as a function of the carrier density $N_{\mathrm{s}}$ theory and experiment - results of Ref. [1] (points) and Ref. [10] (triangles). Upper and lower curve as in Fig. 2.

the dominating elastic scattering mechanism is an isotropic one. Possible scattering mechanisms could be alloy scattering or scattering due to the interface roughness.

The presented theory can be also applied to interpreting experimental results of other authors $[4,8]$. The main difference is that all existing antilocalization data were taken for heterojunctions and not quantum wells. In the case of heterojunctions $\left\langle k_{z}^{2}\right\rangle$ depends much stronger on the carrier density. On the base of the presented theory we calculated $H_{\mathrm{so}}\left(N_{\mathrm{s}}\right)$ for the case of heterojunctions. Comparison of the theoretical results with experimental data of Ref. [4] and [8] is shown in Fig. 3 .

\section{Conclusion}

Spin relaxation in $2 \mathrm{D}$ systems was studied. Theoretical approach taking into account spin relaxation according to different precession movements with their 
appropriate relaxation times was presented. We show that if all elements are properly taken into account one can get good consistent quantitative (up to order of magnitude) description of the all existing experimental data without any fitting parameters. This allows to state that the spin precession as described by the Dyakonov and Perel model is the main spin relaxation mechanism in the 2D systems with no inversion symmetry.

\section{Acknowledgments}

We would specially thank M. Dyakonov and V. Perel for many advices, help and illuminating discussions. We would like also thank B. Jusserand, B. Etienne, T. Dietl for useful discussions.

\section{References}

[1] W. Knap, C. Skierbiszewski, E. Litwin-Staszewska, F. Kobi, A. Zduniak, J.L. Robert, G.E. Pikus, S.V. Iordanskii, V. Mosser, K. Zekentes, Phys Rev. B, in publication.

[2] S. Hikami, A.I. Larkin, Y. Nagaoka, Prog. Theor. Phys. 63, 707 (1980).

[3] P. Pfeffer, W. Zawadzki, Phys. Rev. B 41, 1561 (1990).

[4] P.D. Dresselhaus, C.M.M. Papa vassillion, K. Sacks, Phys. Rev. Lett. 68, 106 (1992).

[5] E. Litwin-Staszewska, F. Kobbi, M. Kamal-Saadi, D. Dur, C. Skierbiszewski, H. Sibari, K. Zekentes, V. Mosser, A. Raymond, W. Knap, J.L. Robert, Solid State Electron. 37, 665 (1994).

[6] W. Knap, D. Dur, A. Raymond, C. Meny, J. Leotin, S. Huant, B. Etienne, Rev. Sci. Instrum. 63, 3293 (1992).

[7] G.E. Pikus, V.A. Maryshehan, A.N. Titkov, Fiz. Tekn. Poluprovodn. 22, 185 (1980) [Sov. Phys.-Semicond. 22, 115 (1980)].

[8] J.E. Hansen, R. Taboryski, P.E. Lindelof, Phys. Rev. B 47, 16040 (1993).

[9] M.I. Dyakonov, V.Yu. Kachorovskii, Sov. Phys.-Semicond. 20, 110 (1986).

[10] G.E. Pikus, A.N. Titkov, in: Optical Orientation, Ed. F. Maier, B. Zakharchenya, North-Holland, Amsterdam 1984. 